\title{
Competencias y metacompetencias de los periodistas digitales
}

\author{
Competences and meta-competences \\ of digital journalists
}

\footnotetext{
(D) Jorge Cruz

jacruz@puce.edu.ec

Pontificia Universidad Católica del Ecuador (Quito)

https://orcid.org/0000-0002-5327-2152
}

DOI https://doi.org/10.32719/26312514.2020.3.5

\section{Resumen}

El perfil de los periodistas y sus competencias se pueden revisar a la luz de los avances que la tecnología digital ha introducido en la profesión. El presente trabajo se plantea encontrar un modelo de construcción de las competencias y metacompetencias que lidien con elementos cognitivos, personales, sociales y éticos. Sobre el mapa de estas habilidades, se colocarán las visiones de estudiosos de la cultura digital, así como académicos relacionados con el ciberperiodismo. El objetivo será encontrar un mejor entendimiento y una visión fresca sobre las áreas que deben repensarse en la formación y reflexión de los perfiles de los profesionales de la información en el presente y frente a los cambios venideros.

\section{Abstract}

The profile of journalists and their competences can be reviewed in the light of the advances that digital technology has introduced in the profession. This paper aims to find a model for the construction of competencies and meta-competencies that deal with cognitive, personal, social and ethical elements. On the map of these skills the visions of scholars of digital culture, as well as academics related to cyber journalism will be placed. The objective will be to find a better understanding and a fresh vision on which areas should be rethought in the training and consideration of the profiles of journalists in the present and in the face of the coming changes.

\section{Palabras clave · Keywords}

Periodismo; competencias; periodismo digital; perfil; habilidades.

Journalism; competences; digital journalism; profile; skills. 


\section{Introducción}

Decir que el periodismo y los periodistas cambiaron a partir del advenimiento de la tecnología digital no es solo repetitivo sino que, después de casi dos décadas del siglo XXI, es una aseveración que merece varios matices, distintas profundidades y, sobre todo, otras miradas. La tecnología, efectivamente, transforma al periodismo, cuando sea que hayan aparecido los avances. El periodista constantemente se capacitó para utilizar máquinas de escribir, cámaras análogas y/o digitales, teletextos, computadoras personales o teléfonos inteligentes e internet ubicuo. Los profesionales de la información se han adaptado a la tecnología y la han adaptado a su labor, de una manera macluhaniana. Como bien comenta Salaverría $(2017,20)$, el periodismo es "en el fondo y desde sus orígenes, la profesión de los períodos".

El presente trabajo busca reflejar, por un lado, que el profesional del ciberperiodismo y el estudio de su transformación pueden tener una lectura alternativa, más allá de la tecnología que se usa o cómo las relaciones cambian, sino cómo las competencias que poseen los periodistas - o las que les demanda el público- se reestructuran. Por otro lado, se discute cómo la profesión subsiste con varios de sus pilares fundacionales, más allá de la adaptación a la tecnología que rediseña la sociedad.

Para alcanzar este cometido, primero se presentan ciertas visiones de cómo el periodista se (auto) define, cómo sus rutinas se modifican a partir de varias fuentes y cómo se entiende la labor en el siglo digital. A continuación, es necesario trazar un modelo que explique cómo se organizan las competencias de una profesión frente a la búsqueda de objetivos performativos y sociales. Finalmente, a partir de la lectura de varias visiones sobre el nuevo perfil, se propone una mirada integral a las competencias y a las metacompetencias que se desprenden de la relación entre el periodista y el mundo en el que desarrolla su trabajo.

Dentro del decálogo que Bill Kovach y Tom Rosenstiel (2014) armaron en sus Elementos del periodismo, mencionan como séptimo mandamiento que el periodismo debe hacer que el significante sea interesante y relevante. Una manera de entender esta premisa es que el periodista debe comprender qué puede contar con la información que posee, las herramientas que maneja y el entorno en el cual transita, en definitiva, qué tan competente es para contar historias.

Lo importante será encontrar alternativas didácticas para explicar el nuevo perfil del periodista a partir de sus competencias, pero sobre todo tender un puente con los formadores de los nuevos periodistas. Cuando se exponen las nuevas competencias, se desarrolla también una aproximación a posibles resultados de aprendizaje, áreas de estudio y reflexión, y necesidades de la profesión que los centros de educación y capacitación deben tener en cuenta. El documento se plantea como una propuesta inicial para el trabajo en estos aspectos. 


\section{Antecedentes}

Los periodistas pueden trazar el inicio de su profesión en la era moderna hasta las primeras décadas del siglo XX y, sin duda, durante toda su evolución laboral han luchado constantemente con los cambios tecnológicos, para utilizarlos y encontrar nuevos espacios. Desde la llegada de la señal radiofónica, hasta el advenimiento de internet, el periodista y sus audiencias generaron constantes contratos de producción y consumo de contenidos noticiosos; sin embargo, es notorio que, con el advenimiento de espacios digitales ubicuos se marcaron diferencias en el estilo de presentación de historias, los métodos de recolección de información, distribución y modelo de negocio. Zelizer $(2019,344)$ menciona cómo la tecnología impactó en el periodismo.

Perfecciona el enfoque del periodismo en los actos de compresión y extracción, dando nuevas dimensiones a lo que se entiende por tamaño, brevedad, capacidad de búsqueda y capacidad de recuperación. Amplifica la orientación del periodismo a la velocidad y la aceleración, la instantaneidad y la simultaneidad que lo acompañan. Y promete experiencias de interacción cada vez más interactivas y, en muchos casos, nuevos grados de accesibilidad.

El periodista cambia, como cambia la tecnología, pero es claro que el centro de su trabajo (sus valores y rol) se mantiene intacto. Postman (2000) se preguntaba si los avances contribuían con el pensamiento racional y, en caso de la labor de los profesionales de la información, habría que considerar si su acercamiento de dispositivos ayuda a mejorar las condiciones de estos pilares o la espectacularidad más bien puede distraer la atención de los existentes déficits (Zelizer 2019).

El papel actual de los periodistas se define también a partir de la relación con los cambios y cómo la sociedad se apropia de esos cambios; en este sentido, las audiencias digitales pueden ser consideradas como espectadores evolucionados, donde su experiencia es incorpórea y anónima (Garza 2002). El público digital tiene más herramientas para realizar un escrutinio más especializado sobre el contenido. Por ejemplo, al mirar el advenimiento del periodismo ciudadano, es importante tener en cuenta cómo los agregadores de noticias, los motores de búsqueda, los sitios oficiales, se convierten en fuentes que pueden fortalecer la calidad de la información recopilada que, como resultado, puede generar una mayor calidad y profundidad periodística (Kaul 2012; Phillips 2010).

Los reporteros han debido redefinir cuál es su rol y cómo deben variar sus habilidades para contar historias a cada paso de los cambios políticos, económicos sociales e institucionales, en favor de un determinado sistema de medios (Willnat, Weaver y Choi 2013). El rol social de los periodistas puede mirarse desde una visión integradora y fusionada entre: informante, intérprete y defensor (Ward citado en Willnat, Weaver y Choi 2013); pero también se puede disgregar en cuatro roles bien definidos: informante, intérprete, movilizador y opositor al poder (Shoemaker y Reese 2014). En cualquier de los casos, se pueden identificar funciones, autoimpuestas por la deontología misma de la profesión, 
que buscan el bien a partir del trabajo periodístico, que no presentan relación directa con el uso o no de la tecnología en ninguno de los procesos de recolección, producción o difusión de noticias.

Un acercamiento adicional y complementario sobre la labor periodística será fijarse en las dimensiones del periodismo que presenta Hanitzch, ya que el contenido también se verá afectado con el tipo de relación que el periodista tendrá ante algunos factores y actores (Oller et al. 2015). Cada dimensión presentará dos polos opuestos, en los que los periodistas podrán autoreferenciarse. En la primera, el Intervencionismo, el periodista puede considerarse activo o pasivo, en función de cómo se involucra ante los hechos de la noticia; de su participación, acompañado o no de una postura más emocional.

En la segunda dimensión, los profesionales de la información se podrán ubicar en uno u el otro extremo de la orientación al mercado. Aquí, los periodistas pueden desarrollar su labor con más distancia o cercanía a los intereses de los mercados o a los intereses del bien público. Es una dicotomía entre tratar a las audiencias como consumidores o como ciudadanos. Como tercera y final dimensión - la distancia al poder-, Hanitzch plantea una bifuración entre los periodistas leales o adversarios a la autoridad. Esta visión nos muestra a un periodista afectado, postiva o negativamente, a partir de su relación con valores deontológicos, que en primera instancia no plantean una relación o modificación con relación a la cercanía con la tecnología.

El periodista seguirá contrastando fuentes, verificando información y — desde una visión ideal- trabajando en función del bien común y de la mejor toma de decisiones de sus públicos. Con esto manifestado, y con la comprensión de que esta naturaleza no se modificará diametralmente a partir de la tecnología, también vale la pena revisar algunos elementos que sí se transformaron. Después de todo, las rutinas están dictadas por la tecnología, los plazos, el espacio y las normas (Becker y Vlad citado en Wahl-Jorgensen y Hanitzsch 2009). Salaverría propone entender al ciberperiodismo a partir de una multidimensionalidad, entendida en cuatro enfoques:

El tecnológico, relacionado con el consumo y la producción multiplataforma; el empresarial, caracterizado por la concentración de las empresas informativas; el profesional, cuyo corolario ha sido la polivalencia temática, funcional y mediática de los nuevos roles periodísticos; y, el de los contenidos, cuya convergencia y confluencia deriva en la moderna multimedialidad del lenguaje ciberperiodístico (Salaverría citado en López García y Limia Fernández 2015, 153).

Para ubicar los cambios latentes en la profesión a partir de internet y toda la tecnología digital, se podrán mirar ciertos aspectos en el proceso mismo del trabajo periodístico. Una manera de organizar esta revisión es la que propone Waisbord (2019) al describir estos cambios con las cinco W's y una $\mathrm{H}$, en referencia a las preguntas estructurales del periodismo. Sobre esto, es interesante reflexionar sobre cómo la tecnología digital modi- 
ficó la ubicuidad de la información, su recolección y difusión (dónde, cuándo). Por otro lado, también se han modificado las temáticas posibles (qué) y las razones que pueden encontrar periodistas o públicos para que sean relevantes (por qué). También las formas de recolección de información sin duda se transformaron a partir de las nuevas tecnologías (cómo) y la posibilidad de que más personas se cobijen bajo las ropas del periodista, por ejemplo, los periodistas ciudadanos (quién).

Adicionalmente, una visión sobre la actualidad de los periodistas es que su profesión o carrera ha sufrido la construcción de nuevos perfiles e incluso la visita de personajes de otras áreas, que tienen más posibilidades y recursos narrativos a partir de la adopción de nuevas tecnologías (Deuze y Witschge 2018). Por otro lado, Torzuko (citado en López García 2015) enlista nuevos perfiles profesionales requeridos en salas de noticias en la era digital. Esta descripción evidencia que la palabra periodista podría mirarse como una visión arcaica de una labor en constante movimiento:

1. Planificador de medios sociales

2. Responsable de reputación digital

3. Experto en SEO

4. Experto en SEM

5. Responsable de tráfico online

6. Analista web

7. Arquitecto de la información digital

8. Experto en usabilidad web

9. Gestor de contenidos

10. Desarrollador multimedia

Las bases sociales y la importancia cultural del oficio se adaptan, pero mantiene intacta su naturaleza, mientras que la parte instrumental del periodismo sufrió la latente transformación digital, incluso en los tipos de cargos o perfiles que se pueden encontrar en los entornos laborales. En esta revisión, será interesante mirar estos cambios, a la luz de las competencias que debe tener el periodista, que a su vez tendrán naturalezas con relación a lo social y lo instrumental.

\section{Competencias del periodista}

Para iniciar la revisión, será importante iniciar por una explicación terminológica para, tras esa definición más clara, se buscará entender la construcción y naturaleza de las competencias. En primer lugar, pueden mirarse como la característica de una persona que muestra su disposición a llevar a cabo un desempeño ulterior, esta competencia se construye a partir de conocimientos, habilidades, rasgos o motivos (Hartle, 1995 en Le Deist 
y Winterton, 2005). La generación de competencias, en el campo profesional, supondría tres objetivos particulares, según García Manjón y Pérez López (2008, p. 10):

- Generar un profesional hábil y de buena integración en el mercado laboral.

- Alcanzar una fácil adaptación, adecuación y transición frente a futuras competencias que puedan generarse en el transcurso de la profesión.

- Promover una movilidad hacia ámbitos profesionales diversos.

Adicionalmente, Escudero Muñoz explica -a partir de un planteamiento para la construcción de conocimientos y habilidades en entornos académicos universitarios- a las competencias como la "capacidad de realizar actuaciones inteligentes ante problemas complejos en contextos" (2019, p.10). El trabajo de este teórico además sirve de base para este planteamiento, pues plantea una manera de comprender a las competencias a partir de un mapa (Figura 1), formado por cuatro variables que se interconectan con un eje y que guardan una relación integradora entre sí.

Figura $\mathrm{N}^{\circ} \mathbf{1}$ : Mapa de competencias

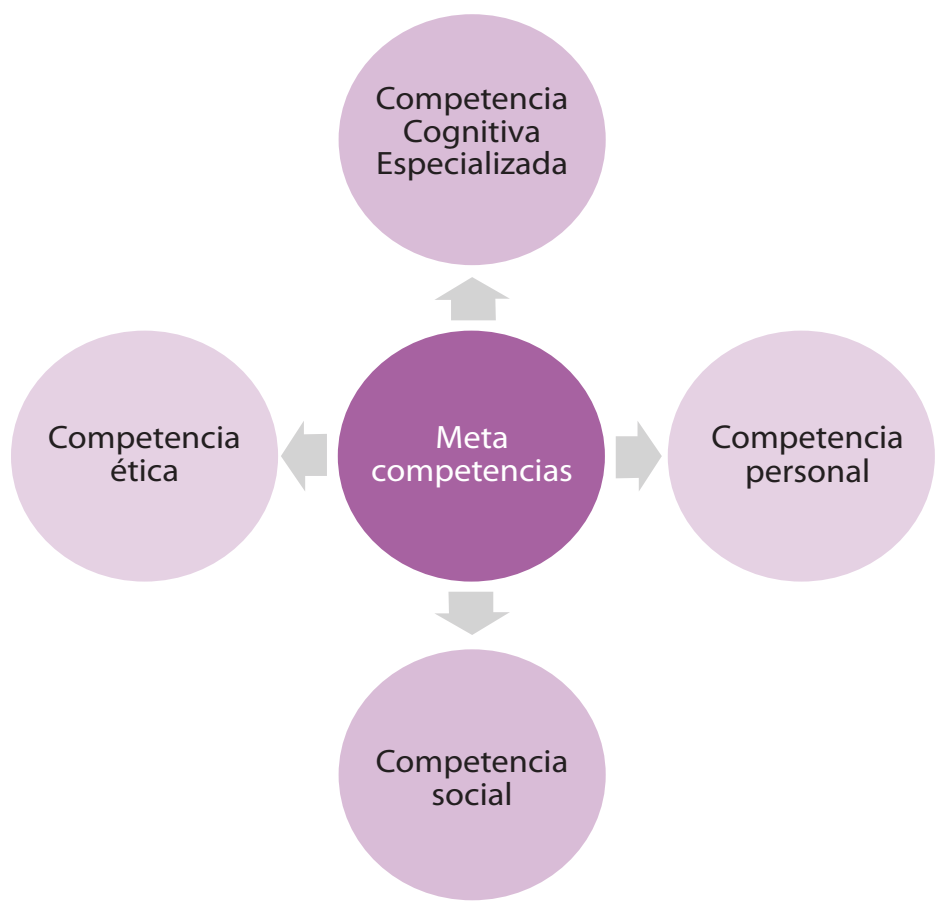

Fuente: Escudero Muñoz 2009. Elaboración propia. 
En primera instancia, para explicar la competencia cognitiva especializada, se debe comprender a esta como la formación científica o académica especializada de una labor o profesión. Serán los conocimientos constitutivos, los procedimientos de trabajo, pero también de gestación y obtención del conocimiento. Es decir, será la manera en que el profesional aprende a aprender el desarrollo de su oficio o carrera.

Escudero Muñoz (2009) plantea que las competencias de conocimiento y las propias de la profesión (las metacompetencias) serán una parte importante, pero no la única que se debe tomar en cuenta en un mapa de competencias. Habla de la necesidad de mirar con atención al desarrollo del manejo autónomo personal y laboral, así como la relación con otros.

Así, un par de aristas necesarias para la construcción de un perfil integral estarán en competencias de reflexión (personales) y de interacción con otro (sociales). Adicionalmente, recalca que "no solo habría que preparar profesionales intelectualmente competentes, humanos y capaces de idear y hacerse responsables con otros de proyectos comunes, sino también profesionales pertrechados de conciencia ética, prestos a someter sus valores y actuaciones a criterios éticos defendibles" (12). Las competencias éticas deben estar presentes al definir lo necesario para nuevos profesionales al momento de una construcción académica o una línea laboral.

Finalmente, las metacompetencias se podrán entender como el desarrollo de ciertas habilidades relacionadas al meta conocimiento de una labor; es decir, lo que se desarrolla en el ejercicio mismo de una profesión, lo que se aprende a partir de la resolución de ciertos problemas y dinámicas laborales. Estas muestran la capacidad humana de autorregular y modificar el aprendizaje mismo por parte de los individuos (Brandsfor et al., citados en Escudero Muñoz 2009).

El mapa es un plano en el cual se podrán colocar algunas visiones sobre cómo se ha construido el ciudadano y el periodista en la era digital; así, se plantea un acercamiento al perfil del ciberperiodista y sus competencias. Es necesario aclarar que este trabajo no busca ser la definición de cómo se construye al profesional de la información en la era digital, pero sí un aporte metodológico y procedimental para que, sobre esta base, se puedan construir nuevas lecturas y más interpretaciones. Esto es justo con la profesión misma, pues, como se revisó anteriormente, los cambios en el periodismo aparecen constantemente y lo importante no serán las determinaciones absolutas, sino encontrar formas de interpretar estas transiciones.

Para construir este modelo de competencias del periodista en la era digital, se escogieron cuatro visiones: Renee Hobbs, una autoridad en alfabetización mediática; Howard Rheingold, un ensayista sobre las implicaciones de la tecnología en la cultura, la política y la sociedad; ambas recogidas en el estudio de Proxémica digital, de John A. McArthur (2016). Estas perspectivas plantean las características de un usuario digital común. Para complementarlas, se revisan los postulados de dos estudiosos de la comunicación y el pe- 
riodismo y la naturaleza del cambio en la profesión que proponen Neil Thurman y Xosé López García. A continuación, se revisarán estas perspectivas y, posteriormente, se las distribuirá a partir del modelo de Escudero Muñoz.

La visión de Hobbs plantea que la información y el contenido son elementos trascendentales para revisar la manera en que se entiende la cultura digital. Adicionalmente, plantea la revisión de las acciones y las relaciones en línea. Plantea que es necesario enfrentar una alfabetización digital y mediática; a partir de ella, se pueden revisar las nuevas relaciones, costumbres y lenguajes. Este análisis se evidencia en cinco habilidades para la era digital, planteadas como competencias (McArthur 2016, 125):

1. Acceder y comprender información e ideas compartidas digitalmente

2. Analizar la información en línea para determinar su calidad y credibilidad

3. Crear contenido multimodal a través de formas de medios

4. Reflexionar sobre las acciones de uno en el entorno en línea

5. Actuar de manera socialmente responsable como miembro de una comunidad.

Rheingold, por su parte, se plantea cinco necesidades para el desarrollo de la vida en el siglo XXI. El ensayista construye estos parámetros a partir del término capacidad, que puede entenderse como la oportunidad, lugar o medio para ejecutar algo, según la Real Academia Española (RAE). Esta perspectiva se plantea a partir de acciones sobre el consumo mediático y la forma de relacionarse con la información:

1. La capacidad de dirigir y enfocar la atención personal.

2. La capacidad de informar, persuadir o comprometerse con otros.

3. La capacidad de trabajar con otros hacia un objetivo común.

4. La conciencia de la red, la capacidad de interactuar con las redes sociales, tecnológicas y educativas.

5. El consumo crítico, la capacidad de reconocer información creíble cuando la vemos.

En el primer acercamiento de la visión periodística, Thurman $(2015,17)$ señala seis características que los periodistas requieren en la época digital. Los "proveedores de noticias", como los determina Thurman, enfrentan retos relacionados a los diferentes pasos de la labor periodística, que pasa por la recolección, selección, producción y difusión de la información:

1. Centrarse en las necesidades específicas de su audiencia.

2. Adaptarse al estilo y la estructura del medio digital.

3. Ser más selectivos sobre la innovación, basando las decisiones en evidencias. 
4. Evaluar continuamente las asociaciones comerciales y tecnológicas para asegurar que son en el mejor interés de la organización.

5. Evitar gestos simbólicos y la mala ejecución en favor de desarrollos bien diseñados.

6. Invertir en contenido original, en la investigación y el desarrollo.

Finalmente, López García (2015) se plantea un par de competencias, que se desprenden de su relectura a los elementos periodísticos de Kovach y Rosenstiel. El académico español apunta que (1) la verificación es una característica vital para el buen desarrollo profesional del periodista. Por otro lado, señala que los periodistas cuentan con una cualidad que se refuerza con el desarrollo del ciberperiodismo. López García afirma que los profesionales de la información (2) suelen presentarse de manera más modesta ante sus deberes y objetivos profesionales.

Antes de poner en consideración la organización de las competencias, es necesario mencionar que esta propuesta busca una visión ontológica y formativa, por lo que no están expresadas competencias que podrán relacionarse con herramientas o dinámicas específicas, como la del manejo del lenguaje y la narrativa. Sin embargo, sí es una arista que debe mirarse en futuras investigaciones. Por otro lado, la manera de contar historias se podrá encontrar - con las modificaciones propias de las plataformas- de forma similar a pesar de los dispositivos que se utilicen para los distintos pasos del ejercicio de los profesionales de la información:

La mezcla, la hibridación y la convergencia de medios - que caracteriza al momento actual del periodismo- evidencian lo inútil de buscar divisiones temporales para referirse a momentos de realce de uno u otro invento mediático (Cruz, Cevallos y Yépez-Reyes 2018, 71).

A continuación, la tabla 1 presenta la organización de las competencias en función de lo planteado por Escudero Muñoz. Como se podrá revisar, todos los campos contaron con aportes de más de un teórico, lo que permite una visión mejor construida, que apunta 
a la integralidad.

Tabla 1: Cuadro de competencias del periodista digital

\begin{tabular}{|c|c|}
\hline $\begin{array}{c}\text { Tipo } \\
\text { de competencias }\end{array}$ & Competencias \\
\hline $\begin{array}{l}\text { Competencias } \\
\text { cognitivas } \\
\text { especializadas }\end{array}$ & $\begin{array}{l}\text { Acceder y comprender información e ideas } \\
\text { compartidas digitalmente } \\
\text { La capacidad de reconocer información creíble cuando la } \\
\text { vemos. }\end{array}$ \\
\hline $\begin{array}{l}\text { Competencias } \\
\text { personales }\end{array}$ & $\begin{array}{l}\text { Reflexionar sobre las acciones de uno en el entorno en } \\
\text { línea } \\
\text { La capacidad de dirigir y enfocar la atención personal }\end{array}$ \\
\hline $\begin{array}{l}\text { Competencias } \\
\text { sociales }\end{array}$ & $\begin{array}{l}\text { Actuar de manera socialmente responsable como } \\
\text { miembro de una comunidad } \\
\text { La capacidad de interactuar con las redes sociales, } \\
\text { tecnológicas y educativas } \\
\text { La capacidad de trabajar con otros hacia un objetivo } \\
\text { común } \\
\text { Centrarse en las necesidades específicas de su } \\
\text { audiencia }\end{array}$ \\
\hline $\begin{array}{l}\text { Competencias } \\
\text { éticas }\end{array}$ & $\begin{array}{l}\text { Evaluar continuamente las asociaciones comerciales y } \\
\text { tecnológicas para asegurar que son en el mejor interes } \\
\text { de la organización. } \\
\text { Modestia frente a sus responsabilidades y retos con la } \\
\text { sociedad. }\end{array}$ \\
\hline Metacompetencias & $\begin{array}{l}\text { Analizar la información en línea para determinar su } \\
\text { calidad y credibilidad } \\
\text { Crear contenido multimodal a través de formas de } \\
\text { medios } \\
\text { La capacidad de informar, persuadir o comprometerse } \\
\text { con otros } \\
\text { Adaptarse al estilo y la estructura del medio digital } \\
\text { Ser más selectivos sobre la innovación, basando las } \\
\text { decisiones en evidencias } \\
\text { Evitar gestos simbólicos y la mala ejecución en favor } \\
\text { de desarrollos bien diseñados. } \\
\text { Invertir en contenido original, en la investigación y el } \\
\text { desarrollo. } \\
\text { La verificación, a partir de técnicas nacidas con el uso } \\
\text { de las nuevas tecnologías }\end{array}$ \\
\hline
\end{tabular}

Fuente: Escudero Muñoz 2009; Hobbs, 2010 (rojo); López García 2015 (negro); Rheingold 2010 (verde); 


\section{Conclusiones}

Sobre la construcción del modelo, es interesante revisar cómo todos los campos en el mapa de Escudero Muñoz tienen elementos identificables en las propuestas de Hobbs.

López García, Rheingold y Thurman son los teóricos con los que se trabaja para una mejor comprensión de cómo el modelo puede funcionar para definir un perfil de periodista en la era digital. Vale la pena comentar y detenerse en cada una de las competencias, particularmente en las sociales y las metacompetencias.

Como se puede apreciar, las competencias cognitivas, personales y éticas se relacionan con acciones que bien podrían mencionarse en entornos análogos y en perspectivas relacionadas sobre la base misma de la profesión. Es particularmente importante revisar la competencia propuesta por López García: "Modestia frente a sus responsabilidades y retos con la sociedad".

La tecnología digital ofrece oportunidades similares, en un primer nivel, a todos sus usuarios. La capacidad de publicar inmediatamente información y de encontrar maneras profesionales de hacerlo genera un sisma en las bases mismas del periodismo. Así, los profesionales de la información han sentido su papel cada vez más ligero en la sociedad. López García habla de la modestia, pues si bien la valía de los periodistas está más allá de la discusión, su actitud frente a las relaciones y posiciones que ocupa se deben revisar para encontrar un valor actualizado y siempre importante.

Son las competencias sociales las que pueden generar una discusión más profunda, pues internet creó una manera nueva de relacionarse y socializar, no solo entre sujetos, sino entre el público y el contenido. Esto se puede evidenciar particularmente en la competencia propuesta por Rheingold: "La capacidad de interactuar con las redes sociales, tecnológicas y educativas" y la de Thurman: "Centrarse en las necesidades específicas de su audiencia”.

Estas competencias exigen que el periodista se interconecte con el resto de los usuarios de la tecnología digital para poder recabar información y presentarla, pero sobre todo para comprender cómo es que se comportan y cuáles son los deseos de estos públicos. Va más allá de complacer a la gente; es revitalizar y reinventar parte de lo que se ha llamado canónicamente "olfato periodístico".

Por otro lado, Rheingold señala que es necesaria "la capacidad de informar, persuadir o comprometerse con otros". Este compromiso es sin duda el puente que, en jornadas llenas de información o infoxicación, se puede tender entre los periodistas y los públicos, en función de crear contenido para la sociedad y a partir de sus reales intereses.

Es necesario detenerse ahora en el análisis de las metacompetencias, pues -como 
explica Escudero Muñoz- se trata del área que se desarrolla en el centro mismo de la profesión y su desempeño. Hobbs señala que analizar la información en línea para determinar su calidad y credibilidad es un requerimiento de los ciudadanos digitales, pero se vuelve trascendental para los periodistas que, a partir de la verificación, no solo confirman la credibilidad de su información, sino que fortalecen la suya entre los públicos. Esto se relaciona con lo propuesto por López García sobre las oportunidades que ofrecen las nuevas tecnologías para llevar a cabo dicha comprobación.

Hobbs también hace referencia a la creación de contenido multimodal como una habilidad necesaria. Si bien los ciudadanos pueden hacerlo con las herramientas disponibles, los periodistas son, por naturaleza, relatores de historias y deben mantenerse atentos no solo a los nuevos dispositivos, sino a las narrativas que traen consigo para poder mantener el contenido "interesante y relevante" como señalan Kovach y Rosenstiel. Sobre esto, Thurman habla de cómo el periodista debe adaptarse al estilo y la estructura del medio digital.

Las narrativas obedecen a la historia y la manera en la que se quiera contar, pero también debe existir una comprensión de dónde se contará y de las posibilidades y alcance del canal y medio en el que se hará.

Thurman, al tratarse de un académico relacionado directamente con los cambios en el periodismo, es quien más aportó al campo de las metacompetencias. En primera instancia, menciona a la selectividad sobre la innovación y la mejor toma de decisiones a partir de evidencias.

Esto expone cómo el profesional de la información deberá ir más allá de las tecnologías y de lo vistoso de las presentaciones que la era digital ofrece y mantenerse atento a lo que las evidencias pueden proporcionar a su trabajo.

Por otro lado, invita a trabajar en desarrollos bien diseñados que permitan ir más allá de la espectacularidad o la superficialidad y proponga una mejor presentación de la información y mejor ejecución de la labor periodística. Adicionalmente, señala que será importante apoyar el contenido original, su investigación y desarrollo. En una hora de información "recalentada", es importante que el periodista digital vaya más allá de replicar, retuitear o repostear, y tenga presente la voluntad del trabajo propio.

Este análisis se propone como una visión inicial hacia un objetivo ulterior, la mejor comprensión de la formación del perfil de los periodistas de los medios contemporáneos. Si bien esta visión debe complementarse con elementos pedagógicos, metodológicos y directrices que decanten en resultados de aprendizaje, será importante empezar a revisar cómo las competencias de los periodistas pueden ofrecer luces para el trabajo de académicos, profesores, capacitadores y periodistas que deseen emprender actualizaciones constantes a sus habilidades y potencialidades.

La tecnología digital debe permitir trazar una importante línea de trabajo en las profesiones que sufren impacto a partir de nuevos dispositivos y prácticas en todas sus 
fases. El periodismo necesita repensarse desde su relación con otros, con los temas, pero también sobre la naturaleza de su profesión, su desempeño y las posibles dificultades que puedan aparecer a partir de los cambios. Las competencias y, particularmente, las metacompetencias permiten mirar esta radiografía y detectar las potencialidades y las oportunidades que el sector presentará para el desarrollo personal y profesional de sus practicantes.

\section{Referencias}

Cruz, Jorge, Patricio Cevallos y Verónica Yépez-Reyes. 2018. “Desafíos del periodismo inmersivo". En La comunicación en la nueva sociedad digital, editado por Mónica López Golán, Francisco Campos Freire, Paulo López López y Franco Rivas Echeverría, 65-78 Mérida: Universidad de los Andes.

Deuze, Mark y Tamara Witschge. 2018. "Beyond journalism: Theorizing the transformation of journalism”. Journalism 19 (2), 165-88.

Escudero Muñoz, Juan. 2009. "Las competencias profesionales y la formación universitaria: posibilidades y riesgos”. Revista de Docencia Universitaria (16): 65-82.

Garza, Gilbert. 2002. “The internet, narrative, and subjectivity”. Journal of Constructivist Psychology 15 (3): 185-203.

García Manjón, Juan Vicente y María del Carmen Pérez López. 2008. “Espacio europeo de educación superior: competencias profesionales y empleabilidad”. Revista Iberoamericana de Educación 46 (9): 4 .

Hobbs, Renee. 2010. "Digital and Media Literacy: A Plan of Action". A White Paper on the Digital and Media Literacy Recommendations of the Knight Commission on the Information Needs of Communities in a Democracy. Washngton: Aspen Institute.

Kaul, Vineet. 2012. “Journalism in the age of digital technology”. Romanian Journal of Communication and Public Relations 14 (1): 59-72.

Kovach, Bill y Tom Rosenstiel. 2014. The Elements of Journalism: What Newspeople Should Know and The Public Should Expect. Nueva York: Three Rivers.

Le Deist, Françoise Delamare y Jonathan Winterton. 2005. “What is competence?”. Human Resource Development International 8 (1): 27-46.

López García, Xosé. 2015. "La renovación de los elementos del periodismo”. En Periodismo en cambio, editado por Xosé López García y Francisco Campos Freire, 186-207. Porto: Media XX- Publishing, Research and Consulting.

López García, Xosé y Moisés Limia Fernández. 2015. “Los nuevos perfiles profesionales en el ecosistema digital”. En Periodismo en cambio, editado por Xosé López García y Francisco Campos Freire, 148-70 Porto: Media XX- Publishing, Research and Consulting.

McArthur, John. 2016. Digital Proxemics: How Technology Shapes The Ways We Move. Nueva York: 
Peter Lang.

Oller, Martín, Palmira Chavero, Julia Carrillo y Patricio Cevallos. 2015. “La autopercepción de los roles profesionales de los periodistas en Ecuador”. Quórum 12 (1): 157-87.

Phillips, Angela. 2010. “Old sources: new bottles”. En New Media, Old News Journalism \& Democracy in the Digital Age, 2.a ed., editado por Natalie Felton, 87-101. Londres: Sage.

Postman, Neil. 2000. “The humanism of media ecology”. Proceedings of the Media Ecology Association 1 (1): 10-6.

Rheingold, H. 2010. "Attention, and other 21st-century social media literacies". Educause Review 45 (5): 14 .

Salaverría, Ramón. 2017. “Tipología de los cibermedios periodísticos: bases teóricas para su clasificación". Revista Mediterránea de Comunicación/Mediterranean Journal of Communication 8 (1): 19-32.

Shoemaker, Pamela J. y Stephen D. Reese. 2014. Mediating The Message in The 21st Century. Nueva York: Routledge.

Thurman, Neil. 2015. "Journalism, gatekeeping, and interactivity". En A Handbook of Digital Politics, editado por Stephen Coleman y Deen Freelon, 357-74. Cheltenham: Edward Elgar.

Waisbord, Silvio. 2019. “The 5Ws and 1H of digital journalism”. Digital Journalism 7 (3): 351-8.

Wahl-Jorgensen, Karin, y Thomas Hanitzsch. 2009. The Handbook of Journalism studies. Nueva York: Routledge.

Willnat, Lars, David H. Weaver y Jihyang Choi. 2013. "The global journalist in the twenty-first century". Journalism Practice 7 (2): 163-83.

Zelizer, Barbie. 2019. "Why journalism is about more than digital technology". Digital Journalism 7 (3): $343-50$ 